\title{
VEGF blockade decelerates the growth of a murine experimental osteosarcoma
}

\author{
DEZHEN YIN ${ }^{1,2}$, TANGHONG JIA ${ }^{1}$, WEIMING GONG ${ }^{1}$, HAIYING YU ${ }^{2}$, PAUL H. WOOLEY ${ }^{2}$, \\ MICHAEL P. MOTT ${ }^{2}$ and SHANG-YOU YANG ${ }^{2}$ \\ ${ }^{1}$ Shandong University School of Medicine, Jinan 250010, P.R. China; ${ }^{2}$ Department of Orthopaedic Surgery, \\ Wayne State University, Detroit, MI 48201, USA
}

Received March 12, 2008; Accepted May 5, 2008

DOI: 10.3892/ijo_00000004

\begin{abstract}
Retrovirus-mediated sFlt-1 gene modification was performed to examine the influence of VEGF in controlling the growth of an experimental osteosarcoma in mice. Human osteosarcoma G-292 cells were in vitro infected with retroviral vectors encoding soluble Flt-1 or LacZ gene before transplanted into proximal tibiae of immune deficient SCID mice to establish experimental orthotopic osteosarcoma. Daily observation and biweekly microCT were performed to monitor tumor development and progression till sacrifice at 8 weeks after tumor cell inoculation for histological and molecular analyses. Successful transgene expression was confirmed in the culture media of sFlt-1 transduced G-292 cells using ELISA, and with positive X-gal staining of the LacZ transduced cells. Noteworthy tumors were grown in all mice on the tibiae receiving G-292 cell inoculation, with clear detection on microCT images starting 2 weeks after inoculation. Over the time period, tumors derived from sFlt-1 transduced G-292 cells were distinctively smaller in size when compared to the ones from wide-type G-292 and G-292-LacZ cells. Histology showed typical osteosarcoma characteristics including severe cellular pleomorphism, bone erosions, and neo-vascularization. Real-time polymerase chain reaction indicated significantly higher sFlt-1 expression in sFlt-1 transduced groups than the wild-type G-292 or LacZtreated groups. Strong expression of oncogenes c-myc and c-fos were also obvious, along with the expression of VEGF in the primary tumor tissue. Overall, data suggest that retrovirus-mediated sFLT-1 gene modification decelerates the osteosarcoma tumor growth in this murine model.
\end{abstract}

Correspondence to: Dr Shang-You Yang, Department of Orthopaedic Surgery, Wayne State University, UHC-7C, 4201 St. Antoine Blvd., Detroit, MI 48201, USA

E-mail: syang@wayne.edu

Key words: osteosarcoma, sFlt-1, VEGF blockage, gene transfer, mouse model

\section{Introduction}

Osteosarcoma is the most common malignant tumor seen in orthopaedic surgery, with high morbidity in young adults and adolescents. Despite intensive treatment, including adjuvant chemotherapy, wide excision of tumors and amputation of the diseased limbs, approximately half of such patients die within 5 years (1). On this basis, it is clear that novel therapeutic approaches are needed for improved osteosarcoma treatment.

One recent novel interventional strategy for cancer is based upon interruption of tumor angiogenesis (2-8). Indeed, angiogenesis plays a critical role in neoplastic processes and is essential for growth, invasion and metastasis of solid tumors (9-11). Among many factors regulating angiogenesis, vascular endothelial growth factor (VEGF) may be one of the most potent (12-14). Enhanced VEGF gene expression has been identified in a number of malignant tumors from breast, lung, ovarian, liver and colon cancer in comparison with normal tissue (15). Specifically, an association between high-level VEGF and poor prognosis of osteosarcoma has been reported $(15,16)$. Using a newly developed murine osteosarcoma model (17), we demonstrated that high VEGF expression clearly associated with the fast tumor growth and early lung metastasis. This recognition leads to potential strategy to target the VEGF pathway as a prospective antiosteosarcoma therapy.

VEGF exerts its biological effects on endothelial cells by binding to its cell surface receptors. Receptors identified to bind VEGF include Flt-1 (fms-like-tyrosine kinase-1, or VEGFR-1) and Flk-1 (fetal liver kinase-1, or VEGFR-2). These VEGF receptors are essential components of signal transduction pathways that affect cell proliferation, differentiation, migration, and metabolism. Soluble Flt-1 is an endogenously expressed, alternatively spliced form of the Flt-1 VEGF receptor $(18,19)$. This form of Flt-1 binds to VEGF with the same affinity and equivalent specificity as that of the full-length receptor, but this binding does not initiate signal transduction because sFlt-1 lacks its intracellular tyrosine kinase domains and the cell association. Indeed, there are numbers of studies trying to apply the sFlt-1 gene transfer technique to halt growth and metastasis of different solid tumors such as lung cancer (20), renal cell 
carcinoma (21), gastric cancer $(22)$, liver cancers $(23,24)$, thyroid carcinoma (25), and ovarian carcinoma (26). Generally, these studies have established the concept that gene therapy may offer a technical means to realize the potential benefits of antiangiogenesis approaches.

In this experiment, we investigated the in vitro transduction efficiency of retroviral vectors encoding sFlt-1 to osteosarcoma G-292 cells and evaluated the effects of sFlt-1 modification on the tumor growth of a murine orthotopic osteosarcoma.

\section{Materials and methods}

Animals. Severe combined immunodeficient (SCID) mice at four weeks of age were obtained from the Jackson Laboratory (Bar Harbor, Maine) and used as hosts for the experimental sarcoma. The animals were housed in a pathogen-free environment and given free access to autoclaved chow and water. All mice were quarantined for one week prior to experimentation.

Osteosarcoma cell lines. Human osteosarcoma cell lines, G-292 (CRL-1423) were obtained from American Type Culture Collection (Manassas, VA) and processed according to the vendor's instructions. G-292 cells were cultured at $37^{\circ} \mathrm{C}$ in a $5 \% \mathrm{CO}_{2}$ incubator, in McCoy's 5a medium with $1.5 \mathrm{mM}$ L-glutamine and $2.2 \mathrm{~g} / 1$ sodium bicarbonate, also contained $10 \%$ fetal bovine serum (FBS), $100 \mathrm{U} / \mathrm{ml}$ penicillin, and $100 \mu \mathrm{g} / \mathrm{ml}$ streptomycin. When $90 \%$ confluence was reached, culture medium was removed, the cell layer rinsed thoroughly with phosphate-buffered saline (PBS), and enzymatically dissociated by adding $0.25 \%$ (w/v) trypsin- $0.03 \%$ (w/v) EDTA. Cells were maintained by subculture at a ratio of $1: 8$. Prior to implantation, the cell suspensions were diluted with $0.5 \%$ $(\mathrm{w} / \mathrm{v})$ trypan blue in $0.16 \mathrm{~mol} / \mathrm{l}$ ammonium chloride to assess cell viability and number.

Construction of retroviral vectors and gene transduction. The original retroviral vector coding for sFlt-1 was kindly provided by Dr Hairong Peng at University of Pittsburgh (27). The titer of the viral vectors was estimated to be $1-2 \times 10^{6} \mathrm{cfu} / \mathrm{ml}$ by limiting dilution. Retrovirus coding for bacteria LacZ was provided from the laboratory of Dr Paul Robins at University of Pittsburgh. For in vitro gene transduction, $500 \mu 1$ of $10^{6} \mathrm{cfu}$ retrosFlt-1 (or MFG-LacZ) was added onto G-292 cells with $500 \mu 1$ of fresh medium including polybrene at final concentration of $8 \mu \mathrm{g} / \mathrm{ml}$. Cells were incubated at $37^{\circ} \mathrm{C}$ for $8 \mathrm{~h}$ before the second addition of the same amount of the retroviral vectors. The cells were then maintained at $37^{\circ} \mathrm{C}$ incubator with $5 \% \mathrm{CO}_{2}$. The incorporation of sFlt-1 in genetic DNA of the transduced cells in the culture media was evaluated by ELISA. LacZ transgene expression was detected using X-gal staining. G-292 cells transduced with sFlt-1 or LacZ were transplanted in proximal tibiae of the SCID mice according to the experimental design.

Enzyme-linked immunosorbent assay (ELISA). sFlt-1 transgene expression in the culture medium collected from G-292 cell cultures were assessed using Quantikine ELISA kits (R\&D Systems) with a pair of rabbit anti-human sFlt-1 antibodies
(R\&D Systems) according to the manufacturer's instructions and the standardized protocol previously described (28).

$X$-gal staining. $\mathrm{X}$-gal staining was performed to confirm LacZ gene transduction, as previously described (29). Briefly, the cells were fixed in the fixative ( $4 \%$ formaldehyde, $0.5 \%$ glutaraldehyde, $0.1 \mathrm{M} \mathrm{Na}$ phosphate buffer, $\mathrm{pH}$ 7.2) for $30 \mathrm{~min}$ at room temperature (RT) and washed three times in $0.1 \mathrm{M}$ PBS (pH 7.4) for $5 \mathrm{~min}$, followed by incubating overnight in $\mathrm{X}$-gal staining solution $[1 \mathrm{mg} / \mathrm{ml}$ of 5 -bromo-4-chloro3-indolyl $\beta$-D-galactoside (X-gal), $2 \mathrm{mM} \mathrm{MgCl}_{2}, 5 \mathrm{mM}$ potassium ferrocyanide, $5 \mathrm{mM}$ potassium ferricyanide, $0.01 \%$ sodium deoxycholate, and $0.02 \%$ Nonidet-P40]. The cells were washed the next day and evaluated for the intensity and distribution of the blue coloration under a light microscope.

Establishment of the orthotopic osteosarcoma. The Institutional Animal Investigation Committee approved all animal procedures. SCID mice were randomly divided into 3 groups with 10 animals per group, to receive wide-type G-292 cells, sFlt-1 transduced cells, and LacZ-transduced cells, respectively. All mice were anesthetized by i.p. injection of a mixture of xylazine $(8 \mathrm{mg} / \mathrm{kg})$ and ketamine $(100 \mathrm{mg} / \mathrm{kg})$. Under strict sterile conditions, a $0.5-\mathrm{cm}$ incision along the lateral collateral ligament of the knee was made to expose the proximal of tibia. A $0.8-\mathrm{mm}$ dental drill was used to drill a small hole across the metaphysis. The wound was rinsed with PBS containing penicillin $\mathrm{G}(500 \mathrm{U} / \mathrm{ml})$ and streptomycin $(500 \mu \mathrm{g} / \mathrm{ml})$ before closing the skin cut by simple interrupted sutures. Culture medium (50 $\mu \mathrm{l})$ containing $10^{6}$ of wild-type or gene modified osteosarcoma cells were injected into the hole immediately after surgery. The other limb received a sham operation without injection of tumor cells.

All animals were monitored throughout the study with daily visual inspection for general health and tumor development. Animals were sacrificed at 8 weeks after tumor cell innoculation by $\mathrm{CO}_{2}$ asphyxiation. Legs containing orthotopic tumors were harvested for histological and molecular evaluations.

MicroCT evaluation. An eXplore Locus MicroCT system (GE Medical Systems, London, ON, Canada) was used to monitor the tumor growth and characteristics of bone lesions. Mice were scanned immediately following tumor cell implantations, and every 2 weeks thereafter. All mice were fully anesthetized $(10 \mathrm{mg} / \mathrm{kg}$ of xylazine and $120 \mathrm{mg} / \mathrm{kg}$ of ketamine) and restrained during each CT scanning. Scan parameters were set at $45 \mu \mathrm{m}$ isotropic voxel size, 400 projections, 400-ms exposure time, $80 \mathrm{~kW}$ voltages, and $450 \mu \mathrm{A}$ current. The length and width of tumor were measured on microCT images using the GE HC MicroView ${ }^{\circledR}$ software with Analysis ${ }^{+}$Version 1.0 (GE Healthcare) (Fig. 4D and E). The tumor volumes $(V)$ were calculated using the formula of $V=L \times W^{2} \times 0.52$, where $L$ is the length and $W$ represents the width of the tumor (30).

RNA extraction and real-time quantitative PCR for gene expression. Primary osteosarcoma tissues including the adjacent bone were snapped-frozen in liquid nitrogen at the 
time of sacrifice. A portion of the tumor-bone powder homogenized in $0.5 \mathrm{ml}$ of TRIzol solution (Gibco BRL) using a glass grinder pestle. Total RNA extraction was performed using a commercial kit (Tel-Test Inc., Friendswood, TX) in accordance with the manufacturer's instructions. The precipitated RNA was then treated with DNase and passed through a spin column (Rneasy mini kit, Qiagen) for further purification. Reverse transcription and real-time PCR for the expression of VEGF, sFlt-1, c-myc, and c-fos was performed using the ABI PRISM 7700 Sequence Detector (PE-Applied Biosystems), as detailed previously (31). The comparative gene expressions of the experimental tumor groups over the sham controls were calculated according to the formula given in the manufacturer's manual (32).

Histology process and immunohistological (IHC) examination. Proximal tibiae bearing osteosarcoma tissue and receiving sham operation were collected. Tissues were fixed in buffered formalin, decalcified in 12\% EDTA and embedded in paraffin at consistent orientation. The bone tumors in tibia were cut longitudinally and other tissues were cut in multiple layers. All tissues were stained with hematoxylin and eosin, and examined under a Zeiss light microscope. Digital photomicrographs were captured and analyzed using Image-Pro Plus analysis software (Media Cybernetics, Silver Spring, $\mathrm{MD)}$.

IHC was performed on primary orthotopic tumor sections to detect the expression levels of VEGF, sFlt-1 according to the vendor's instructions and the protocol published previously (33). Briefly, paraffin sections were deparaffinized in xylene, rehydrated in graded alcohols and water. Hydrogen peroxide $(0.3 \%)$ was applied to diminish endogenous peroxidase followed by microwave incubation to enhance the antigens. After blocking with $1.5 \%$ normal goat serum for $1 \mathrm{~h}$, the sections were incubated overnight with the primary antibodies (2 $\mu \mathrm{g} / \mathrm{ml}$, BD Pharmingen) in a moisturized chamber at $4^{\circ} \mathrm{C}$. Biotin-conjugated secondary antibody and avidin-biotin enzyme reagents were sequentially applied for $30 \mathrm{~min}$ between extensive washes. The color was developed by adding 3,3'diaminobezidine tetrahydrochloride (DAB). In negative control sections, an irrelevant antiserum was applied at the same concentration as the primary antibody. Digital images were captured and analyzed using the Image-Pro software package. The level of positive staining and localization was evaluated in six different fields, and expressed as integrated optical density (IOD). The comparison of the IODs among groups was carried out using the Image-Pro analysis tool.

Statistical analyses. A total of 30 mice were used for this study. Statistical analysis between different groups was performed by the Student's t-test, or the ANOVA test; with the Schafer formula for post hoc multiple comparisons, using the SPSS software package (SPSS; Chicago, IL). A p <0.05 was considered as significant difference. Data are expressed as mean \pm standard deviation.

\section{Results}

Transgene expression. The expression of sFlt-1 protein levels in the culture medium was measured using ELISA. Extensive

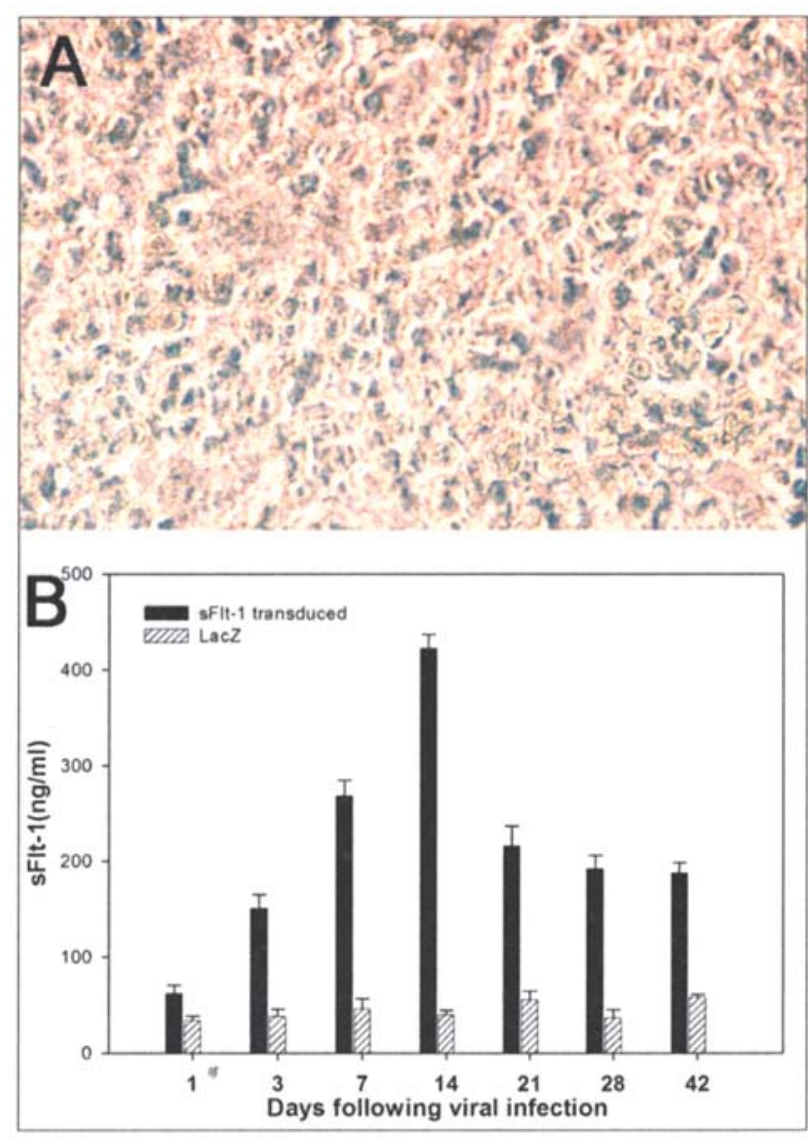

Figure 1. Osteosarcoma cells were in vitro transduced with Retro-sFlt-1 or LacZ. X-gal staining was performed to reveal the successful transduction of LacZ (A, x100). Culture media were collected periodically for detection of sFlt-1 expression using ELISA and summarized as B.

elevation of sFlt-1 levels was detected $24 \mathrm{~h}$ after viral infection and reached peak levels at 14 days (Fig. 1B). The elevated transgene product was maintained for at least 6 weeks in culture. Experimental tumors retrieved from SCID mice with sFlt-1 modified G-292 cell inoculation revealed strong positive immunohistochemical sFlt-1 staining (Fig. 2D). Quantification analysis of sFlt-1 expression using a computerized image analysis system indicated significantly stronger positive stains in sFlt-1 transduced groups in comparison with non-viral controls (wide-type G-292) or LacZ transduced groups (Fig. 3). X-gal staining on LacZ-transduced cells exhibited strong blue color, indicating the successful transfer and expression of $\beta$-galactosidase gene, transduction efficiency was $86 \%$ (Fig. 1A).

Inhibitory effects of sFlt-1 on growth of experimental osteosarcoma. The orthotopic osteosarcoma developed at the proximal tibiae after tumor cell transplantation (Fig. 4A). Macroscopic assessment at sacrifice revealed that the tumors with sFlt-1 gene modification were significantly smaller than the ones derived from wide-type osteosarcoma G-292 cells (Fig. 4B). Periodical microCT scans dynamically monitored the development and growth of the primary tumor and proved a useful monitoring technique in this study (Fig. 4C-G). Analyses of microCT data using MicroView program indicated that all mice developed orthotopic bone tumors at 2 weeks 


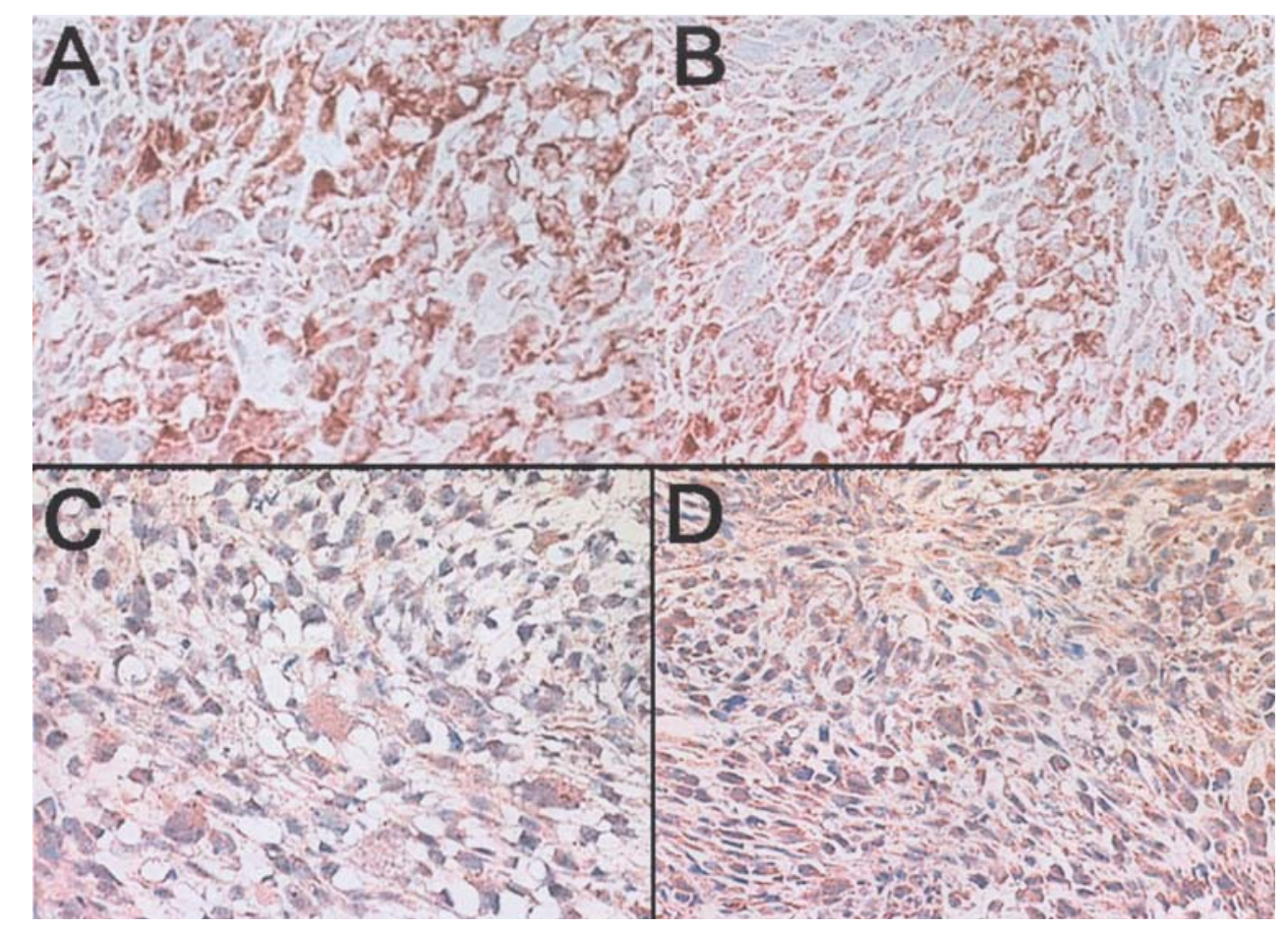

Figure 2. Immunohistochemical stains against human VEGF (A and B) and sFlt-1 (C and D) were performed on the experimental osteosarcoma tissues. Micrographs A and C are tumors derived from the LacZ transduced group; B and D are samples from the sFlt-1 transduced group (magnification: A, x400; B, x400; C, x200; D, x200).

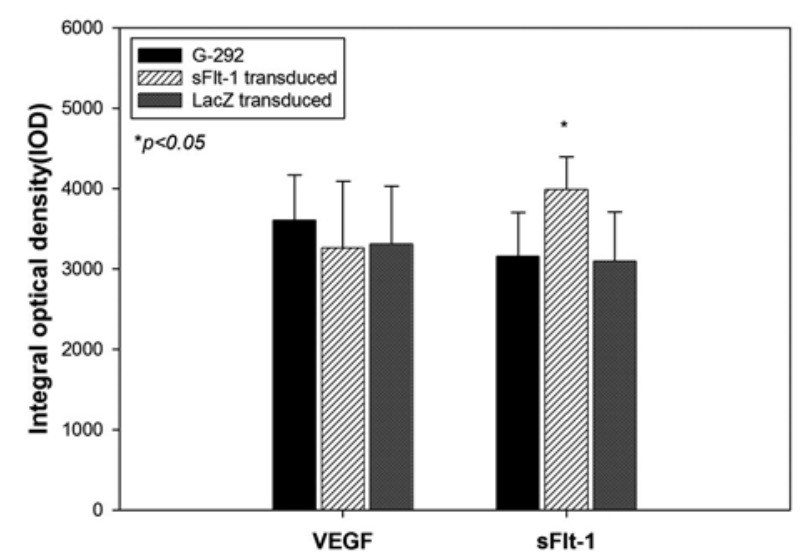

Figure 3. The expression levels of VEGF and sFlt-1 on the osteosarcoma in different groups, quantified as integrated optical density (IOD).

after tumor cell inoculation and the individual tumor volume among groups was not significantly different. At 4,6 , and 8 weeks, however, the average tumor volumes of the nonmodified G-292 and LacZ transduced groups appeared significantly larger than those of sFlt-1 transduced group (Fig. 5). All tumors on the CT images exhibited mixed osteoblastic and osteolytic changes and there was no significant difference among the groups.

Histological and immunohistochemical assessment. Histology showed typical osteosarcoma characteristics including severe cellular pleomorphism, bone erosions, and neovascularization at 8 weeks after tibia primary tumor establishment (Fig. 6).
Immunohistochemical staining illustrated strong positive VEGF staining on all the experimental tumor sections (Fig. 2A and B). However, the experimental tumors derived from the cells with sFlt-1 gene modification exhibited dramatically more transgene expression against sFlt-1 antibody (Fig. 3, $\mathrm{p}<0.05)$.

Molecular assessment. RNA samples isolated from the orthotopic tumors were examined for the gene expression of VEGF, sFlt-1, and oncogenes c-fos and c-myc. Fig. 7 summarizes the comparative gene expressions among groups. Data show strong expression of oncogenes c-myc and c-fos along with the expression of VEGF in the primary tumor tissue. Significantly higher sFlt-1 expression in sFlt- 1 transduced groups was obvious in comparison with G-292 or LacZ-treated groups (Fig. 7, p<0.05).

\section{Discussion}

Strategies to inhibit neoangiogenesis represent a potentially powerful approach for treatment of neoplastic disease. Furthermore, virus-mediated gene modification may offer a potential therapeutic approach with the aspects of sitespecific and long-term effectiveness. In the present study, we attempted to examine the hypothesis that abrogation of the VEGF influences the growth of osteosarcoma, evaluating the retroviral vector-mediated sFlt-1 gene therapy in controlling the osteosarcoma tumor growth in a murine model.

We have previously described a murine orthotopic osteosarcoma model to study progression and metastasis of osteosarcoma and found that high VEGF expression correlates with the early establishment, rapid tumor growth, and the development of pulmonary metastasis (17). It appears that 


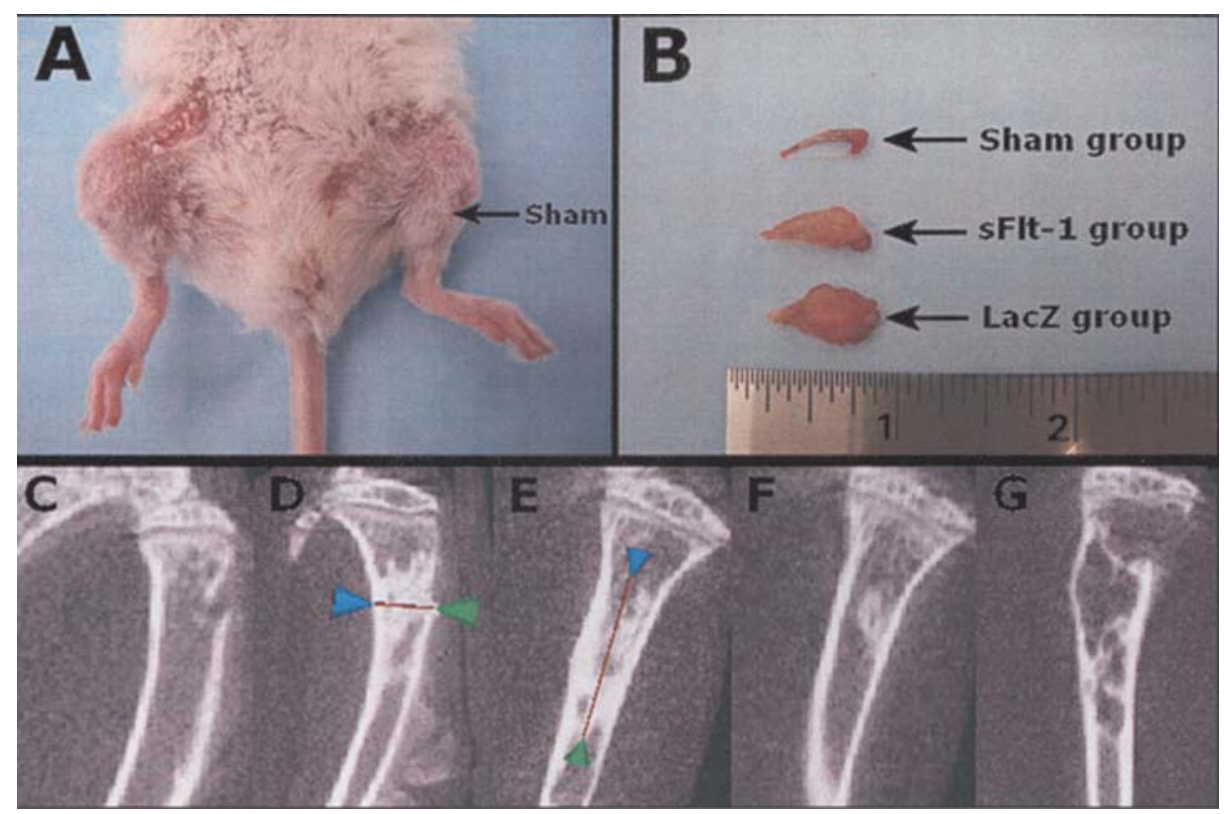

Figure 4. Representative macroscopic views of the experimental tumors at 8 weeks after cells inoculated (A and B) and microCT images of the orthotopic osteosarcoma model. C, Sham operation group; D, Two weeks after wild-type G-292 inoculation; E, Four weeks after wild-type G-292 cell inoculation; F, Two weeks after sFlt-1 modified G-292 cell inoculation; G, 8 weeks after sFlt-1 modified G-292 cell inoculation. Lines on D and E are the measurements for the width and length of the tumor.

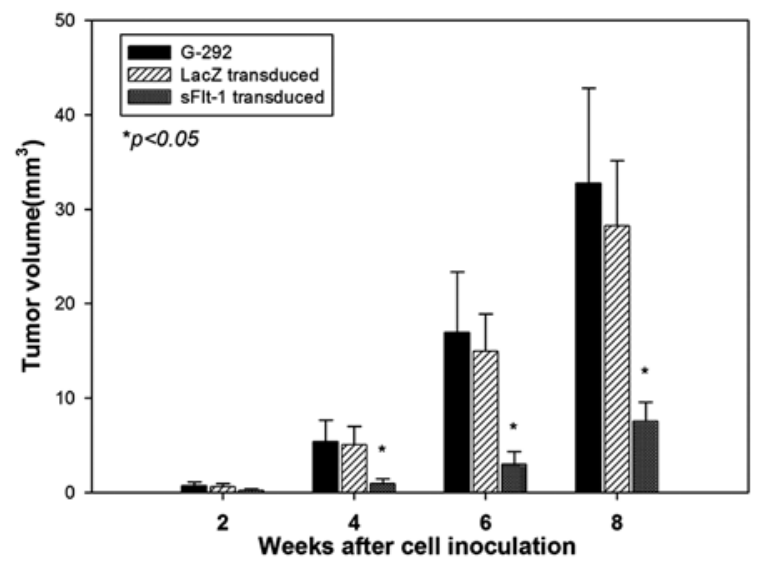

Figure 5. Orthotopic tumor sizes over the experiment period were evaluated on the microCT images. Tumor volumes were calculated using the formula $V=L \times W^{2} \times 0.52$, and data are presented as mean volume $\pm \mathrm{SE}(\mathrm{n}=10$, $\left.{ }^{*} \mathrm{p}<0.05\right)$.

anti-angiogenic therapy, especially the blockade of VEGF effects may be a novel and potentially promising strategy for osteosarcoma. The soluble receptor of VEGF, sFlt-1 is a potent and selective inhibitor of VEGF. sFlt-1 inhibits the angiogenic action of VEGF in two ways. Not only does it bind and sequester VEGF from its biological angiogenic action, but sFLT-1 also forms inactive heterodimers with the extracellular ligand-binding region of the membrane spanning Flt-1 and KDR/Flk-1 VEGF receptors, thereby blocking the phosphorylation and activation of downstream signal transduction pathways for endothelial cell proliferation. In this study, we first established a stable sFlt-1 expressing osteosarcoma cell line by ex vivo transduction of sFlt-1 gene using retroviral vectors into high VEGF-expressing osteosarcoma G-292 cells. This sFlt-1 expressing osteosarcoma

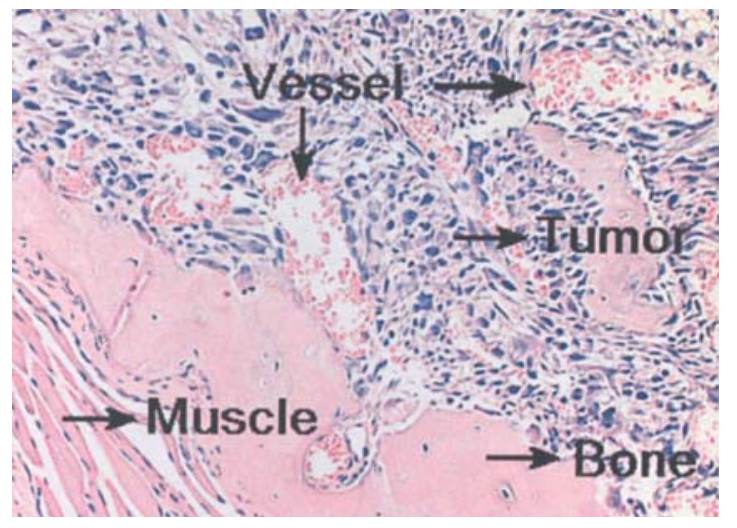

Figure 6. Histological appearance of the experimental osteosarcoma shows typical osteosarcoma characteristics including severe cellular pleomorphism, bone erosions, and neo-vascularization at 8 weeks after tibia primary tumor establishment (H\&E staining; magnification x200).

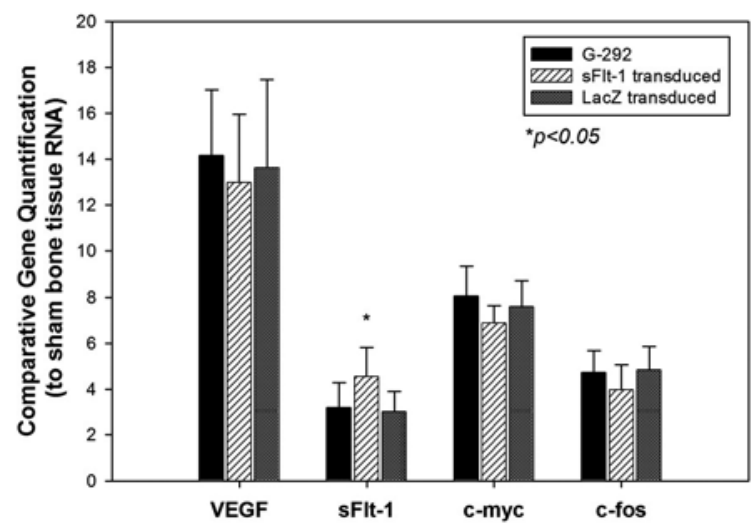

Figure 7. Comparative gene expression quantification using sham operated bone tissue as base controls. Data are expressed as the relative expressions over those from sham bone tissues. Significantly higher sFlt-1 expression in sFlt-1 transduced groups compared with G-292 or LacZ-treated groups $\left({ }^{*} \mathrm{p}<0.05\right)$. 
cells were then transplanted into tibiae of SCID mice to compare with wild-type G-292 cells and LacZ-transduced controls in the tumor development and growth in situ.

Indeed, there are a number of studies applying sFlt-1 gene transfer technique to halt growth and metastasis of different solid tumors such as lung cancer (20), renal cell carcinoma (21), gastric cancer $(22)$, liver cancers $(23,24)$, thyroid carcinoma (25), and ovarian carcinoma (26). Generally, the administration of sFlt-1 gene inhibited the neo-vascularization and promoted tumor cell apoptosis. However, the therapeutic effects using adenoviral vector was rather transient and some cytotoxicity was reported (34). In our study, human osteosarcoma cell line G-292 cells were transduced with retroviral vectors encoding sFlt-1 before transplanted into the host proximal tibia. It appears that the gene transfer was successful, and the transgene product was detected for at least 6 weeks before and on animal investigations. No acute cytotoxicity in cell culture was noted following 2 times viral infection.

In our results, the development of osteosarcoma was markedly suppressed in the sFlt- 1 group, the average tumor volumes significantly smaller than G-292 and LacZ transduced groups at 4, 6, 8 weeks. It appears that the soluble Flt-1 may be an excellent candidate of therapeutic agents for the longer and effective inhibition of angiogenesis during tumor development/progression. There was no significant difference in the tumor volumes among groups at early development of the experimental tumors ( 2 weeks after cell inoculation), suggesting lesser influence of VEGF in the early tumor development. It is well accepted that the growth of solid tumors greater than 1-2 $\mathrm{mm}^{3}$ is critically dependent on angiogenesis (9).

With the recent expansion of molecular biology techniques, genetic alterations associated the development and metastases of malignant tumors have been observed. Cellular oncogenes have been found to be activated by DNA rearrangements (proviral insertions, chromosome translocations and DNA amplifications). These alterations may result in an increased or deregulated gene expression. Numerous oncogenes and tumor suppressor genes have been identified in osteogenic sarcomas, c-myc and c-fos seem to be expressed with a relative high incidence (35-39). C-myc proto-oncogene on chromosome 8 encodes a transcription factor, which is involved in the regulation of cell growth, DNA replication, and transcriptional regulation of specific target genes (40). $\mathrm{C}$-fos proto-oncogene, the cellular homologue of $\mathrm{v}$-fos, is involved in osteoblast and chondrocytes differentiation (38). Studies have indicated that c-myc and c-fos were overexpressed in the relapsed osteosarcoma and the metastasis cases (36). In G-292 and G-292-LacZ experimental osteosarcoma model, these oncogenes were markedly expressed in the tumor tissues supplementing the high VEGF expression. In sFlt-1 transduced groups, c-myc and c-fos were found with lower expression compared with G-292 or LacZ-treated groups, the difference was not significant.

In the present study, sFlt-1 gene modification appears to decelerate the tumor growth compared with G-292 or LacZtreated groups. However, it could not completely halt the tumor growth and progress. This suggests that angiogenesis is a complex mechanism and attributes to multiple factors and pathways. Blocking the action of a single potent angiogenic factor may not result in complete abrogation of angiogenesis and tumor progression; combination regimes of antiangiogenic approaches with chemotherapy may prove more potent therapeutic efficacy.

\section{Acknowledgements}

This work was supported in part by a grant from Karmanos Cancer Institute, Michigan, and the Fund for Medical Research and Education, Department of Orthopaedic Surgery, Wayne State University.

\section{References}

1. Rosen G, Suwansirikul S, Kwon C, Tan C, Wu SJ, Beattie EJ Jr and Murphy ML: High-dose methotrexate with citrovorum factor rescue and adriamycin in childhood osteogenic sarcoma. Cancer 33: 1151-1163, 1974.

2. Brower V: Tumor angiogenesis-new drugs on the block. Nat Biotechnol 17: 963-968, 1999.

3. Kong HL, Hecht D, Song W, Kovesdi I, Hackett NR, Yayon A and Crystal RG: Regional suppression of tumor growth by in vivo transfer of a cDNA encoding a secreted form of the extracellular domain of the flt-1 vascular endothelial growth factor receptor. Hum Gene Ther 9: 823-833, 1998.

4. Millauer B, Shawver LK, Plate KH, Risau W and Ullrich A: Glioblastoma growth inhibited in vivo by a dominant-negative Flk-1 mutant. Nature 367: 576-579, 1994.

5. O'Reilly MS, Holmgren L, Shing Y, et al: Angiostatin: a novel angiogenesis inhibitor that mediates the suppression of metastases by a Lewis lung carcinoma. Cell 79: 315-328, 1994.

6. O'Reilly MS, Boehm T, Shing Y, et al: Endostatin: an endogenous inhibitor of angiogenesis and tumor growth. Cell 88: 277-285, 1997.

7. Yanase T, Tamura M, Fujita K, Kodama S and Tanaka K: Inhibitory effect of angiogenesis inhibitor TNP-470 on tumor growth and metastasis of human cell lines in vitro and in vivo. Cancer Res 53: 2566-2570, 1993.

8. Yokoyama Y, Dhanabal M, Griffioen AW, Sukhatme VP and Ramakrishnan S: Synergy between angiostatin and endostatin: inhibition of ovarian cancer growth. Cancer Res 60: 2190-2196, 2000

9. Folkman J: Tumor angiogenesis: therapeutic implications. N Engl J Med 285: 1182-1186, 1971.

10. Folkman $\mathrm{J}$ : What is the evidence that tumors are angiogenesis dependent? J Natl Cancer Inst 82: 4-6, 1990.

11. Folkman J: The role of angiogenesis in tumor growth. Semin Cancer Biol 3: 65-71, 1992.

12. Folkman J and Klagsbrun M: Angiogenic factors. Science 235: 442-447, 1987.

13. Kim KJ, Li B, Winer J, Armanini M, Gillett N, Phillips HS and Ferrara N: Inhibition of vascular endothelial growth factor-induced angiogenesis suppresses tumour growth in vivo. Nature 362: 841-844, 1993 .

14. Leung DW, Cachianes G, Kuang WJ, Goeddel DV and Ferrara N: Vascular endothelial growth factor is a secreted angiogenic mitogen. Science 246: 1306-1309, 1989.

15. Lee YH, Tokunaga T, Oshika Y, et al: Cell-retained isoforms of vascular endothelial growth factor (VEGF) are correlated with poor prognosis in osteosarcoma. Eur J Cancer 35: 1089-1093, 1999.

16. Kaya M, Wada T, Akatsuka T, et al: Vascular endothelial growth factor expression in untreated osteosarcoma is predictive of pulmonary metastasis and poor prognosis. Clin Cancer Res 6: 572-577, 2000.

17. Yang SY, Yu HY, Jeffrey E, et al: High VEGF with rapid growth and early metastasis in a mouse osteosarcoma model. Sarcoma 2007: 95628 (7 pages), 2007.

18. Kendall RL and Thomas KA: Inhibition of vascular endothelial cell growth factor activity by an endogenously encoded soluble receptor. Proc Natl Acad Sci USA 90: 10705-10709, 1993.

19. Kendall RL, Wang $G$ and Thomas KA: Identification of a natural soluble form of the vascular endothelial growth factor receptor, FLT-1, and its heterodimerization with KDR. Biochem Biophys Res Commun 226: 324-328, 1996. 
20. Takayama K, Ueno H, Nakanishi Y, et al: Suppression of tumor angiogenesis and growth by gene transfer of a soluble form of vascular endothelial growth factor receptor into a remote organ. Cancer Res 60: 2169-2177, 2000.

21. Yoshimura I, Mizuguchi Y, Miyajima A, Asano T, Tadakuma T and Hayakawa M: Suppression of lung metastasis of renal cell carcinoma by the intramuscular gene transfer of a soluble form of vascular endothelial growth factor receptor I. J Urol 171: 2467-2470, 2004.

22. Sako A, Kitayama J, Koyama H, Ueno H, Uchida H, Hamada H and Nagawa $\mathrm{H}$ : Transduction of soluble Flt-1 gene to peritoneal mesothelial cells can effectively suppress peritoneal metastasis of gastric cancer. Cancer Res 64: 3624-3628, 2004.

23. Graepler F, Verbeek B, Graeter T, et al: Combined endostatin/ sFlt-1 antiangiogenic gene therapy is highly effective in a rat model of HCC. Hepatology 41: 879-886, 2005.

24. Schmidt K, Hoffend J, Altmann A, et al: Transfer of the sFLT-1 gene in Morris hepatoma results in decreased growth and perfusion and induction of genes associated with stress response. Clin Cancer Res 11: 2132-2140, 2005.

25. Ye C, Feng C, Wang S, et al: sFlt-1 gene therapy of follicular thyroid carcinoma. Endocrinology 145: 817-822, 2004.

26. Mahasreshti PJ, Navarro JG, Kataram M, et al: Adenovirusmediated soluble FLT-1 gene therapy for ovarian carcinoma. Clin Cancer Res 7: 2057-2066, 2001.

27. Peng H, Wright V, Usas A, Gearhart B, Shen HC, Cummins J and Huard J: Synergistic enhancement of bone formation and healing by stem cell-expressed VEGF and bone morphogenetic protein-4. J Clin Invest 110: 751-759, 2002.

28. Yang S, Wu B, Mayton L, Evans CH, Robbins PD and Wooley PH: IL-1Ra and vIL-10 gene transfer using retroviral vectors ameliorates particle-associated inflammation in the murine air pouch model. Inflamm Res 51: 342-350, 2002.

29. Sud S, Yang SY, Evans CH, Robbins PD and Wooley PH: Effects of cytokine gene therapy on particulate-induced inflammation in the murine air pouch. Inflammation 25: 361-372, 2001.
30. Attia MA and Weiss DW: Immunology of spontaneous mammary carcinomas in mice. V. Acquired tumor resistance and enhancement in strain A mice infected with mammary tumor virus. Cancer Res 26: 1787-1800, 1966.

31. Yang SY, Wu B, Mayton L, Mukherjee P, Robbins PD, Evans $\mathrm{CH}$ and Wooley PH: Protective effects of IL-1Ra or vIL-10 gene transfer on a murine model of wear debris-induced osteolysis. Gene Ther 11: 483-491, 2004.

32. PE Applied Biosystems: TagMan Cytokine Gene Expression Plate I; Protocol. Anonymous. P/N 4304671, 37-53, 1998.

33. Ben-Josef E, Yang SY, Ji TH, et al: Hormone-refractory prostate cancer cells express functional follicle-stimulating hormone receptor (FSHR). J Urol 161: 970-976, 1999.

34. Mahasreshti PJ, Kataram M, Wang MH, et al: Intravenous delivery of adenovirus-mediated soluble FLT-1 results in liver toxicity. Clin Cancer Res 9: 2701-2710, 2003.

35. Barrios C, Castresana JS, Ruiz J and Kreicbergs A: Amplification of c-myc oncogene and absence of c-Ha-ras point mutation in human bone sarcoma. J Orthop Res 11: 556-563, 1993.

36. Gamberi G, Benassi MS, Bohling T, et al: C-myc and c-fos in human osteosarcoma: prognostic value of mRNA and protein expression. Oncology 55: 556-563, 1998.

37. Pompetti F, Rizzo P, Simon RM, et al: Oncogene alterations in primary, recurrent, and metastatic human bone tumors. J Cell Biochem 63: 37-50, 1996.

38. Van den BS, Rahmsdorf HJ, Herrlich P and Kaina B: Overexpression of c-fos increases recombination frequency in human osteosarcoma cells. Carcinogenesis 14: 925-928, 1993.

39. Wu JX, Carpenter PM, Gresens C, Keh R, Niman H, Morris JW and Mercola D: The proto-oncogene c-fos is over-expressed in the majority of human osteosarcomas. Oncogene 5: 989-1000, 1990.

40. Cole MD and McMahon SB: The Myc oncoprotein: a critical evaluation of transactivation and target gene regulation. Oncogene 18: 2916-2924, 1999. 